
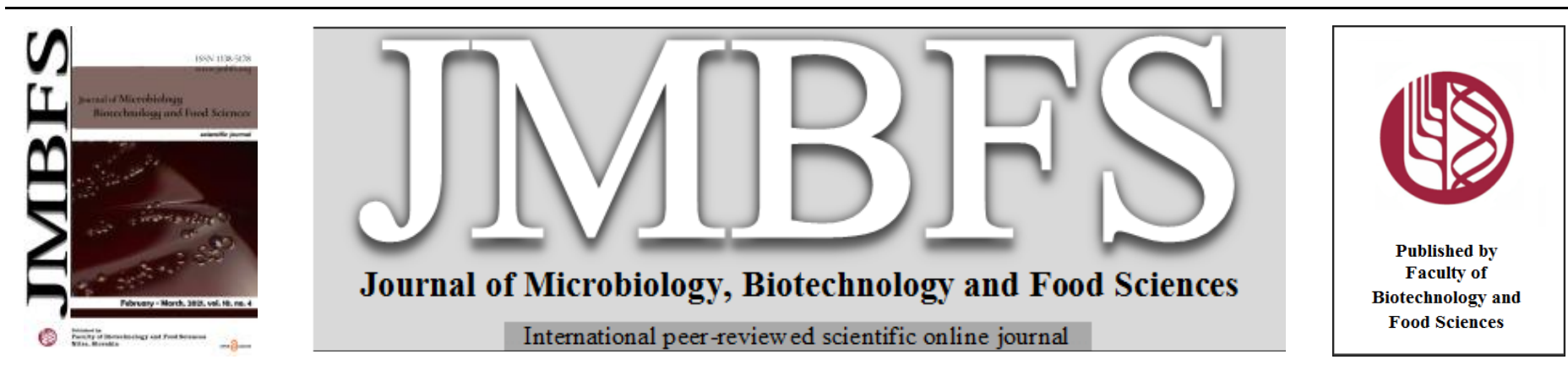

\title{
EVALUATING THE VOLATILE COMPOUNDS DURING ALCOHOLIC FERMENTATION OF DIFFERENT SACCHAROMYCES CEREVISIAE STRAINS IN MIXED JUICES OF CASHEW APPLE AND LONGAN
}

\section{Kanokchan Sanoppa* \\ Address(es): King Mongkut's University of Technology North Bangkok, Faculty of Agro-Industry, Department of Agro-Industry Technology and Management, 25230,} Prachinburi, Thailand.

*Corresponding author: kanokchan.s@agro.kmutnb.ac.th

doi: $10.15414 / j m b f s .2021 .10 .4 .598-603$

\section{ARTICLE INFO}

Received 22. 9. 2019

Revised 12. 10. 2020

Accepted 15. 10. 2020

Published 1. 2. 2021

Regular article open $\mathcal{O}$ access

\begin{abstract}
The purpose of this research was to evaluate the microbiological, chemical, and volatile compounds of mixed juices comprised of cashew apple and longan fermented with two yeast strains of Saccharomyces cerevisiae (C12 and EC1118). The two fermentations in the mixed juices were similar physicochemical parameters (the growth of yeast, $\mathrm{pH}$, total acidity, total soluble solids, and total sugar). The most abundant of the volatile compounds in these wines were ethyl acetate, ethyl hexanoate, ethyl octanoate, ethyl decanoate, isoamyl acetate, ethanol, isobutanol, isoamyl alcohol, 2-phenylethanol, $\beta$-ocimene, and acetic acid. Seven volatile compounds in the wines had odor activity values (OAVs) higher than 1, and also were selected and quantified (ethyl octanoate, ethyl hexanoate, ethyl decanoate, isoamyl acetate, isoamyl alcohol, 2-phenylethanol, and linalool). Ethyl octanoate (fruity, floral, and pineapple) had the highest OAVs in both of the mixed juices fermented with the yeast strains C12 and EC1118. The S. cerevisiae strain EC1118 can significantly produce the highest total volatile contents $(359.23 \mathrm{mg} / \mathrm{L})$, and we also found a higher level of total ester $(95.50 \mathrm{mg} / \mathrm{L})$ in the wines. Additionally, the S. cerevisiae strain EC1118 can provide a higher content of fruity aroma in wines, as indicated by the calculation of OAVs. The sensory analysis of the wines was acceptable to all the panelists, who gave color, clarity, taste, flavor, and overall acceptance scores as "like slightly" to "like moderately."
\end{abstract}

Keywords: Saccharomyces cerevisiae, cashew apple, longan, odor activity values, aroma

\section{INTRODUCTION}

Cashew (Anacardium occidentale Linn var. nanum) is a tropical fruit that was first grown in South America, and then spread to countries such as Thailand, Vietnam, and India (Deenanath et al., 2015; Kaewbutra et al., 2016). The peduncle of the cashew fruit, known as the cashew apple. The composition of the peduncle is rich in tannins, reducing sugars (fructose and glucose), minerals such as calcium, iron and phosphorus, vitamin C, and some amino acids (Pereira and Rodrigues, 2012). It has also been reported to exhibit many medicinal properties, such as antitumor, antimicrobial, and antioxidant activity (Kaewbutra $\boldsymbol{e t} \boldsymbol{a l}$. 2016). Cashew apples can be served fresh or processed as canned fruits, syrup, juice, pickles, jam, chutney, or candy (Gawankar et al., 2018). So, they can serve as a good raw material for wlinemaking. Various compounds have been found during cashew apple wine fermentation, such as ethyl butyrate, ethyl 3methyl butyrate, methyl butyrate, methyl 3-methyl pentanoate, methyl 3-methyl butyrate, and trans-ethyl crotonate (Garruti et al., 2006). However, there have only been a few studies on fermented-beverage products from cashew apples. Longan (Dimocarpus longan Lour) is an important plantation crops in Thailand. It can be used for value-added products such as dried and canned longan. The main nutritional components of dried longan are fructose, sucrose, glucose, amino acids (proline, alanine, aspartic acid, tyrosine, serine, leucine, isoleucine, valine, and glycine), vitamins, and minerals. The major volatile compounds from dried longan include ocimenes, furfural, 5-methyl furfural, benzenemethanol, isoamyl alcohol, 2-furancarboxylic acid ethyl hexadecanoate, and linalool. However, caramelization and the Millard reaction have an effect on the formation of aromatic substances during longan drying process (Chang et al., 1998).

Cashew apple and longan are agricultural products in Thailand, and are suitable to produce wine because they have a rich amount of amino acids and sugar for yeast culture. Cashew is widely cultivated in many regions of Thailand. It has been recognized as one of the new economic plants (Kaewbutra et al., 2016). Longan is interesting to winemaking because it's a processed economic agricultural product in Thailand. Previous studies have also shown that dried longan wine has a pleasant aroma (Sanoppa et al., 2019; 2020). Saccharomyces cerevisiae is one of the most important yeast strains used in winemaking, which play role fermentation in juices and convert sugar ethanol, carbon dioxide, and others compounds and also are tolerant to high ethanol, high sugar, acidic, and anaerobic conditions (Chen and Liu, 2016). S. cerevisiae is one of the yeast strains suitable for producing quality fermented foods and beverages (Carrau $\boldsymbol{e t}$ al., 2008). Yeasts produce secondary metabolites which play as an important wine flavor, including esters, carbonyls, sulfur compounds, and higher alcohols. These are important in determining the aroma and flavor profiles of the wine (Walker and Stewart, 2016)

In this work, the mixed juices of cashew apple and longan were used as raw material for wine fermentation; this had never previously been reported in wine. The aim of this work was to evaluate the chemical, yeast growth and volatile compounds of the mixed juices of cashew apple and longan fermented with two yeast strains of $S$. cerevisiae (C12 and EC1118). This research was to investigate the use of different strains of yeast to aromatic profiles of wine especially, wine that is made from a mixture of fruit. The results will indicate the information to select $S$. cerevisiae strains for aroma profiles formations of wine, and to develop this industry.

\section{MATERIALS AND METHODS}

\section{Microorganism and culture media}

Two strains of Saccharomyces cerevisiae var. bayanus, C12 and EC1118 were obtained from Blue $\mathrm{H}_{2} \mathrm{O}$ Filtration Pty Ltd. (Australia) and Lallemand Inc. (Australia), respectively. The freeze-dried yeasts were propagated in a sterile nutrient broth $(0.25 \% \mathrm{w} / \mathrm{v}$ yeast extract, $2 \% \mathrm{w} / \mathrm{v}$ glucose, $0.25 \% \mathrm{w} / \mathrm{v}$ malt extract, and $0.25 \% \mathrm{w} / \mathrm{v}$ bacteriological peptone at $\mathrm{pH}$ 5.0 ; Y) under static conditions at $25{ }^{\circ} \mathrm{C}$ without agitation for $48 \mathrm{~h}$, and finally stored at $-80{ }^{\circ} \mathrm{C}$ in $\mathrm{YM}$ medium with adding glycerol $20 \% \mathrm{v} / \mathrm{v}$ before use. Yeast was grown in Yeast Malt broth (YM broth) (Difco, USA) and maintained on Yeast Malt agar (YM agar) (Difco, USA).

\section{Substrate preparation and fermentation}

Cashew apples and dried longans were obtained from Thailand, respectively. Dried longan was further dried in a hot-air dryer at $70{ }^{\circ} \mathrm{C}$ for 8 hours. First, 
cashew apples (1 kilogram) were cleaned in tap water. The cashew apples were crushed in a blender and then passed through a filter (The initial Brix of cashew apples juices was $12{ }^{\circ}$ Brix). 100 grams of dried longans were mixed with 300 $\mathrm{mL}$ of distilled water. The sample was homogenized to form longan juice using a blender, and then it was passed through a filter (The initial Brix of dried longans juices was $14{ }^{\circ}$ Brix) (Mohanty et al., 2006; Sanoppa et al., 2019; 2020). The cashew apples juices and dried longans juices were mixed at a ratio of $1: 1(\mathrm{v} / \mathrm{v})$ Then, $200 \mathrm{~mL}$ of the mixed juices of cashew apple and longan were put into 500 $\mathrm{mL}$ Erlenmeyer conical flasks and cotton plugged. Both the cashew apple and longan juices were adjusted to $\mathrm{pH} 3.5$ with $50 \% \mathrm{w} / \mathrm{v}$ malic acid and the Brix adjusted to $20.0 \%$ (The initial Brix of mixed juices was $13{ }^{\mathrm{O}}$ Brix) with pure sucrose, and then $100 \mathrm{ppm}$ of potassium metabisulfite (KMS) was added in the juices (Chen and Liu, 2014; 2016). Fermentations of the sterile mixed juices of cashew apple and longan were conducted under static conditions at $25^{\mathrm{O}} \mathrm{C}$ for 14 days. S. cerevisiae was inoculated with $1 \times 10^{6} \mathrm{CFU} / \mathrm{mL}$.

\section{Microbiological and physicochemical analysis}

During fermentation, wine samples were taken at these intervals: Day 0, 2, 4, 6, $8,10,12$ and 14 , and these samples were stored at freezes $\left(-20{ }^{\circ} \mathrm{C}\right)$ until the analysis was done. Yeast enumeration was done by spread-plating on YM agar. The wines were analyzed $\mathrm{pH}$ value by $\mathrm{pH}$ meter, assessing total acidity conten by titration to the $\mathrm{pH}$ endpoint with $0.05 \mathrm{M} \mathrm{NaOH}$ (A.O.A.C, 2005), total soluble solids ( ${ }^{\circ}$ Brix value) was estimated by a hand refractometer and the phenolsulfuric acid method was used to measure the total sugar content (Agrawal et al., 2015).

\section{Volatile compounds}

The identification of volatile compounds was performed using the headspace solid-phase microextraction (HS-SPME) technique with a Divinylbenzene/Carboxen/Polydimethylsiloxane (DVB/CAR/PDMS) fiber $(50 / 30 \mu \mathrm{m}$, Supelco, Bellefonte, PA, U.S.A.) The determination was performed in combination of Gas chromatography mass spectrometry (GC/MS) (Agilent $7890 \mathrm{~B}$, detector on a DB-Wax UI column. The volatile compounds were performed based on a previously published method by Sanoppa et al. (2019; 2020).

The odor activity values are defined as the ratio between $\mathrm{c}$ is the total concentration $(\mathrm{mg} / \mathrm{L})$ of each volatile compound in the wines, and $\mathrm{t}$ is the odor threshold value $(\mathrm{mg} / \mathrm{L})(\mathrm{OAVs}=\mathrm{c} / \mathrm{t})$ (Cheng et al., 2015).

\section{Sensory analysis}

The sensory attributes of the mixed fruit wines (such as color, clarity, flavor, taste, and overall acceptance) were evaluated using a 9-point Hedonic scale (1=dislike extremely, 2=dislike very much, 3=dislike moderately, 4=dislike slightly, 5=neither like nor dislike, $6=$ like slightly, $7=$ like moderately, $8=$ like very much and 9=like extremely) among 30 panelists (10 women, 20 men, aged 20-45) selected from staff and the postgraduate students in the Faculty of AgroIndustry, King Mongkut's University of Technology North Bangkok who are familiar with wine consumption. The wine samples $(50 \mathrm{~mL})$ were evaluated using transparent glasses which were labeled with random three-digit numbers (Ray $\boldsymbol{e}$ al., 2012). Another set of the mixed fruit wines and grape wine (commercial wine) were evaluated as a second replication the following day (Mohanty $\boldsymbol{e t}$ al. 2006).

\section{Statistical analysis}

All experiments were performed in triplicate. Data are expressed as the mean \pm standard deviation (SD). The results were compared by ANOVA using the SPSS Statistics package version 20.0 at a significance level of $p<0.05$ (SPSS Inc., Chicago, IL). Principal component analysis (PCA) was used to analyze the data of volatile compounds in wines with the software Minitab 18.

\section{RESULTS AND DISCUSSION}

\section{Microbiological and chemical parameters}

During the fermentation of the mix of the cashew apple and longan juices, the two strains of $S$. cerevisiae yeasts exhibited similar yeast population (Fig. 1A) Both strains' yeasts culture achieved yeast population of approximately $1.0 \times 10^{-6}$ $-5.0 \times 10^{-7} \mathrm{CFU} / \mathrm{mL}$, which was maintained until the end of the process. The maximum yeast populations in the wines of strains $\mathrm{C} 12$ and EC1118 were 7.67 and $7.59 \log \mathrm{CFU} / \mathrm{mL}$, respectively, on the $4^{\text {th }}$ day. The growth of yeast in the wines during the first four days of fermentations rapidly increased, and gradually decreased as the wine fermentation time increased.

The chemical compositions of the wines produced by two strains of $S$. cerevisiae are presented in Figures 1B, 1C, 1D, and 1E. As shown in Figure 1E, the $\mathrm{pH}$ of the wines by the two strains increased slightly after fermentation, ranging from pH 3.5-3.9. Similar results in papaya wines were published by Lee et al. (2010;
2013) found in durian wine and Chen and Liu (2014) for lychee wine In addition, the total acid content remained relatively constant during fermentation $(0.11-0.12 \%)$ (Fig 1D), which was similar to previous results reported by Okeke et al. (2015), who found during the fermentation period, the $\mathrm{pH}$ of mixed pineapple and watermelon wine was in the acidic range, which was also similar to research published by Balogun et al. (2017). As the result, the $\mathrm{pH}$ values of the wines were correlated with total acidity values. The total soluble solid decreased initially and then tended to stabilize. The Brix decreased from about $20 \%$ to approximately $9 \%$ for all wines after fermentation (Fig 1C). The Brix value was reduced most quickly in both strains of the $S$. cerevisiae cultures Figure 1E shows the change in total sugar during fermentation in both strains $S$. cerevisiae cultures. The total sugar of the wines decreased slightly during fermentation, from around $6 \%$ to $0.4 \%$. The total sugar value was reduced more quickly in wine fermented with EC1118 than with C12.

(A)

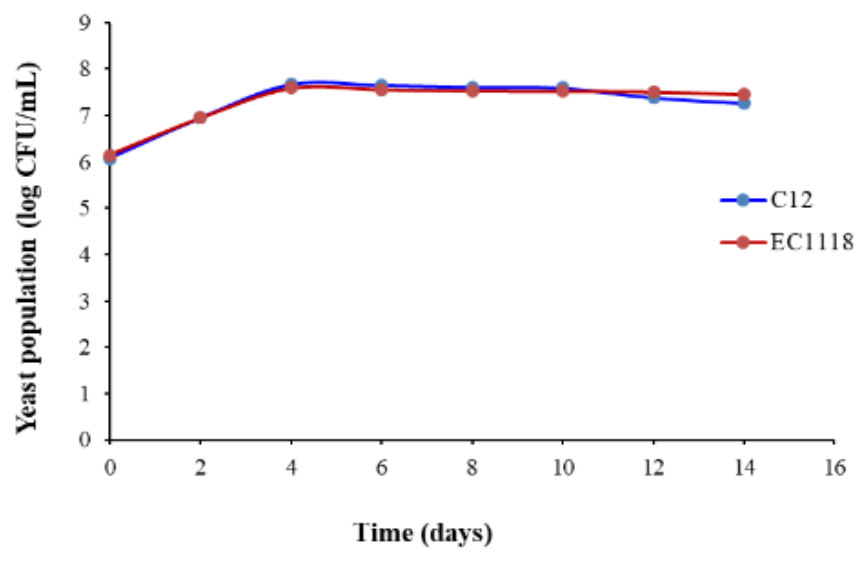

(B)

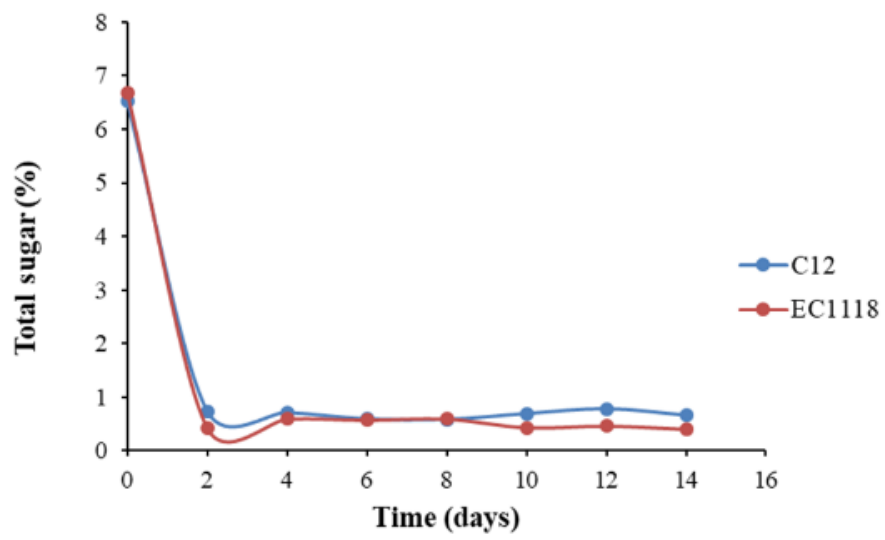

(C)

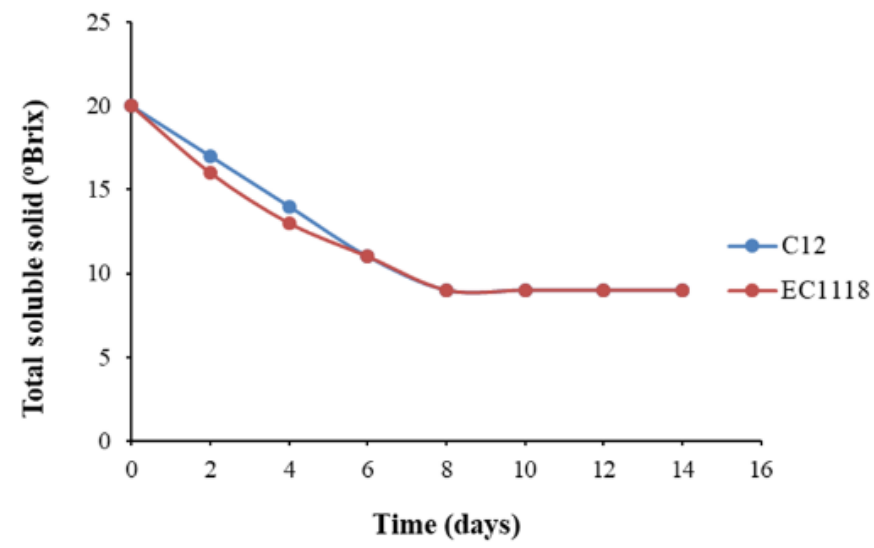


(D)

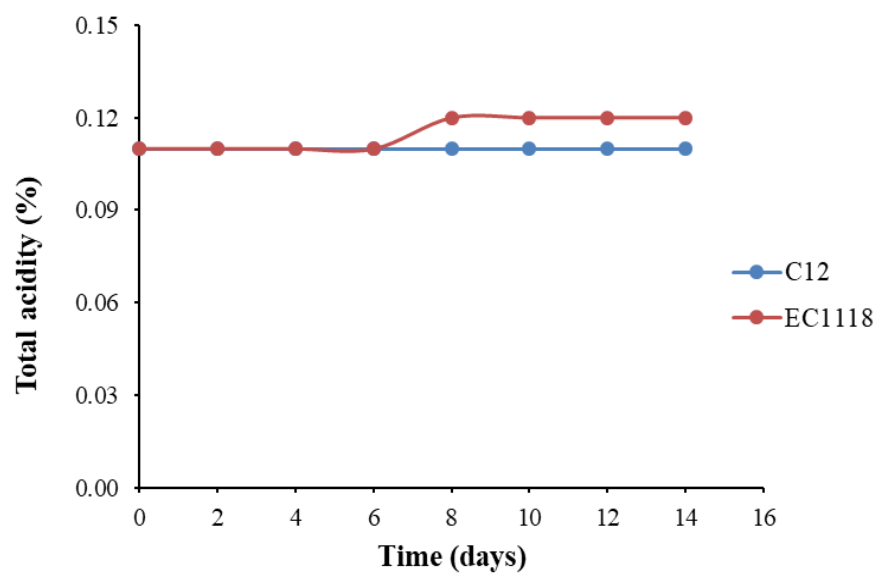

(E)

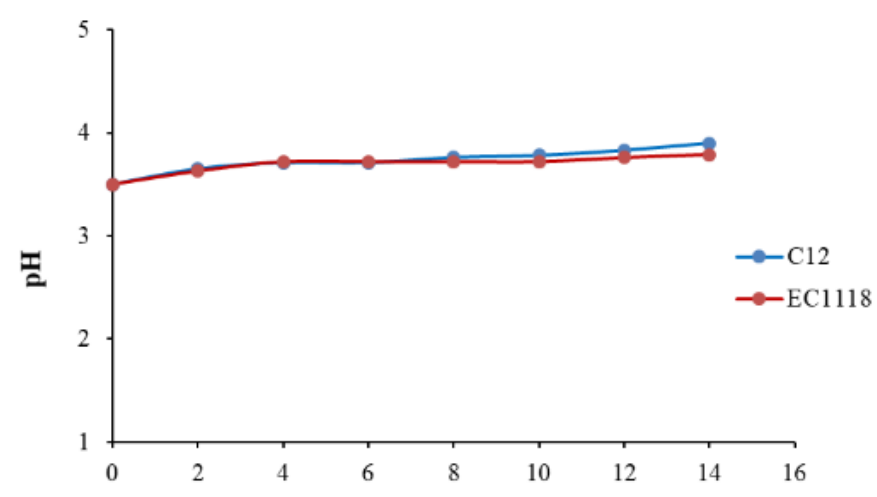

Time (Days)

Figure 1 The growth of yeasts $(\mathrm{CFU} / \mathrm{mL})(\mathrm{A})$, the total sugar content (\%) (B), the total soluble solids ( ${ }^{\mathrm{O}}$ Brix) (C), the total acidity (\%) (D) and $\mathrm{pH}$ value (E) of the mixed juices of cashew apple and longan fermented with two S. cerevisiae yeasts.

\section{Volatile compounds}

We determined the aromatic profiles of wines produced by different yeast strains. During the fermentation of the mixed juices of cashew apple and longan, volatile compounds were produced by yeast. Twenty-one volatile compounds were identified in the wines, including nine esters, five alcohols, three terpenes, one acid, and three others (Table 1). Alcohols comprised the largest group of volatiles in the mixed wines $(240 \mathrm{mg} / \mathrm{L}$ to $260 \mathrm{mg} / \mathrm{L}$ ). Higher alcohols (fusel alcohols) are quantitatively major volatile by yeast, and also can have an aromatic effect in wines. Thus, such alcohols are the most important group of compounds that $S$. cerevisiae produces during fermentation (Swiegers et al., 2005; Parapouli et al., 2020). Ethanol, isobutanol, isoamyl alcohol, $\gamma$-methylmercaptopropyl alcohol, and 2-phenylethanol were the main alcohols produced by the two strains of $S$. cerevisiae. Ethanol was the most abundant of the alcohols in the wines. The ethanol content produced by strain C12 importantly exhibited the highest in the wines. Similar studies have found that the concentration of ethanol in dried longan wines ranged from 130-160 mg/L (Sanoppa et al., 2019; 2020), whereas the alcohol content of cashew apple wine was $5.0 \%(\mathrm{v} / \mathrm{v})$ (Mohanty et al., 2006) In addition, isobutanol, isoamyl alcohol, $\gamma$-methylmercaptopropyl alcohol, and 2phenylethanol can be synthesis by two strains, with no significant differences among the cultures. Isoamyl alcohol and 2-phenylethanol can contribute to the flowery and sweet notes of wine, and isobutanol was detected above their threshold (Zhang et al., 2018). The Ehrlich products of leucine and valine derived from isoamyl alcohol and isobutanol. These are considered as cheap "fusel alcohols", whereas the phenylalanine derived 2-phenylethanol (Swiegers et al., 2005; Etschmann et al., 2008). Higher alcohol synthesis is correlated with $\alpha$-keto acid synthesis. This occurs via the catabolic or Ehrlich pathway or anabolic pathway, which involves amino acids synthesis via their biosynthetic pathway from glucose. The catabolism process of branched-chain amino acid is transaminated to form $\alpha$-keto acid ( $\alpha$-ketoisocaproic acid from leucine; $\alpha$ ketoisovaleric acid from valine; and $\alpha$-keto- $\beta$ methylvaleric acid from isoleucine), and then decarboxylation reaction involves to the synthesis of acids, aldehydes, and alcohols (Dickinson and Norte, 1993; Swiegers et al., 2005). The higher alcohols concentrations are reached, however, are strongly related to the strain used (Carrau et al., 2008).

Esters are the important volatiles in the mixed fruit wines. Most of the esters were synthesized during fermentation from acyl-CoA and alcohol via yeast- alcohol acyltransferase, and this can also can have an effect on the fruity flavors in wine (Swiegers et al., 2005). Esters are catalyzed by an acyl transferase or ester synthase. The reaction requires energy provided by the thioester linkage of the acyl-CoA co-substrate. There are two main groups of flavor-active esters in wines: the ethyl esters and the acetate esters (Saerens et al., 2010). ethyl decanoate, ethyl hexanoate, ethyl octanoate, isoamyl acetate, and 2-phenylethanol were the main esters in the wines (Table 1). Similar results were reported by Zhang et al. (2018), who found five esters above the threshold and also found to be consistent with commercial wine found their esters, which have a potential impact on the aroma profile (Swiegers et al., 2005). Among the two treatments, the mixed juices culture with $S$. cerevisiae EC1118 generated the highest total esters that there was no significant difference among the culture with $S$. cerevisiae $\mathrm{C} 12$ inoculation.

The first group comprises the ethyl esters (the alcohol group is ethanol; the acid group is a medium-chain fatty acid). The biosynthesis of fatty acid ethyl esters (FAEEs) proceeds by alcoholysis or esterification. Alcoholysis mechanism is the synthesis of esters from acylglycerols (alcohols and fatty acyl-CoAs derived from the metabolism of fatty acids) and alcohols. Alcoholysis is important for transferase reaction in which fatty acyl groups from acylglycerols or acyl-CoA derivatives are directly transferred to alcohols. Esterification mechanism is the synthesis of esters from carboxylic acids and alcohols, and is catalyzed by enzymatic mechanisms (FAEE synthases/carboxylesterases) (Saerens et al., 2010). The FAEEs synthesis by mechanism of alcoholysis is catalyzed by enzymatic mechanisms (acyl-CoA:ethanol O-acyltransferases; AEATases), $E E B 1$, and EHT1. ATF1 and ATF2 are not involved in ethyl ester synthesis. $E E B 1$ is the important enzyme for the synthesis of ethyl ester, while $E H T 1$ plays a minor role. However, the deletion of EEBI and $E H T 1$ resulted in a severe decrease in the ethyl ester synthesis (Saerens et al., 2005). Trinh et al. (2010) Depending on the length of the carbon chain of fatty acids, the short-chain ones are more desirable. Studies have shown that the ethyl esters in wines are ethyl heptanoate (grape), ethyl hexanoate (apple), ethyl octanoate (sweet soup), ethyl nonanoate (wax, fruity aroma), ethyl decanoate (floral, soup aroma), and ethyl dodecanoate (sweet, floral, fruity aroma) (Table 1). The concentration of ethyl dodecanoate, ethyl nonanoate, and ethyl heptanoate in the mixed juices cultures with strain C12 had a higher level than in the strain EC 1118. Meanwhile, ethyl octanoate, ethyl decanoate, and ethyl hexanoate produced by yeast strain EC1118 had higher than strain $\mathrm{C} 12$, but all of the esters in the wines showed no significant difference in either of the strains.

The second group is acetate esters (the acid group is acetate, and the alcohol group is ethanol or a complex alcohol derived from amino acid metabolism). The acetate esters are produced at much higher levels, so they can have a more significant impact on wine flavor than ethyl esters (Saerens et al., 2010). The formation of acetate esters is catalyzed by alcohol acetyltransferases, and encoded by the genes $A T F 1$ and $A T F 2$, which use an alcohol and acetyl-CoA as substrates (Swiegers et al., 2005). As seen in Table 1, the acetate esters in wines were isoamyl acetate (banana and pear aroma) and ethyl acetate (fruity, solventlike). The results of acetate esters in all the wines correlated with the alcohol content. The formation of acetate ester dependent on two factors, the concentration of the two substrates (acetyl-CoA and alcohol) and also the activity of the Alcohol acetyltransferase (AATase) (Zhang et al., 2014), which was consistent with the findings in Procopio et al. (2015), who reported acetate esters synthesis that depends on the concentration of their corresponding higher alcohols, except for ethyl acetate, whose synthesis depends on the ethanol concentration. Acetate esters synthesis in S. cerevisiae (isoamyl acetate and ethyl acetate) is ascribed to three enzymes: isoamyl acetyltransferase, ethanol acetyltransferase, and alcohol acetyltransferase (Lilly et al., 2000). The concentration of ethyl acetate and isoamyl acetate were higher in cultured with yeast strain EC 1118, there was no significant difference in the culture with $S$. cerevisiae $\mathrm{C} 12$ inoculation.

Additionally, acids and terpenes were detected in the wines. Terpenes provide many floral, fruity, herbal, and spicy odor in wine, which have low odor thresholds (Zhang et al., 2018). Monoterpenes were synthesized from terpenesynthase. The terpene synthase gene family has expanded in grapevines, which underlines the importance of terpenoids in this species (Ilc et al., 2016) Terpenes, including $\beta$-ocimene, $\alpha$-ocimene, and linalool, were identified in wines (Table 1). $\beta$-ocimene and linalool were found in wine inoculated with yeast $\mathrm{C} 12$ higher level than inoculated with yeast strain EC1118. On the contrary, $\alpha$ ocimene in wines produced by yeast strain EC1118 had higher than for that produced by yeast strain $\mathrm{C} 12$. 
Table 1 Volatile compounds $(\mathrm{mg} / \mathrm{L})$ in mixed juices of cashew apple and longan fermented with two $S$. cerevisiae yeasts.

\begin{tabular}{|c|c|c|c|c|c|c|c|c|}
\hline \multirow{2}{*}{ Compounds } & \multicolumn{2}{|c|}{ Mixed juices } & \multicolumn{2}{|c|}{ S. cerevisiae $\mathrm{C} 12$} & \multicolumn{2}{|c|}{ S. cerevisiae EC1118 } & \multirow{2}{*}{$\begin{array}{l}\text { Odor Threshold } \\
\text { value }(\mathrm{mg} / \mathrm{L})\end{array}$} & \multirow{2}{*}{$\begin{array}{c}\text { Odor } \\
\text { description }\end{array}$} \\
\hline & $\begin{array}{c}\text { Total values } \\
(\mathrm{mg} / \mathrm{L})\end{array}$ & OAVs & $\begin{array}{c}\text { Total values } \\
(\mathrm{mg} / \mathrm{L})\end{array}$ & OAVs & $\begin{array}{c}\text { Total values } \\
(\mathrm{mg} / \mathrm{L})\end{array}$ & OAVs & & \\
\hline \multicolumn{9}{|l|}{ Esters } \\
\hline Ethyl acetate & ND & - & $5.47 \pm 0.12^{\mathrm{NS}}$ & 0.66 & $5.67 \pm 0.94^{\mathrm{NS}}$ & 0.81 & $7.5^{*}$ & $\begin{array}{c}\text { Fruity, } \\
\text { pineapple, } \\
\text { sweet }\end{array}$ \\
\hline Ethyl decanoate & ND & - & $9.19 \pm 1.00^{\mathrm{NS}}$ & 45.95 & $10.24 \pm 0.57^{\mathrm{NS}}$ & 51.20 & $0.2^{* * *}$ & $\begin{array}{l}\text { Fruity, floral, } \\
\text { waxy }\end{array}$ \\
\hline Ethyl dodecanoate & ND & - & $1.98 \pm 0.10^{\mathrm{NS}}$ & - & $1.95 \pm 0.11^{\mathrm{NS}}$ & - & - & - \\
\hline Ethyl heptanoate & ND & - & $2.03 \pm 0.05^{\mathrm{NS}}$ & - & $1.77 \pm 0.07^{\mathrm{NS}}$ & - & - & - \\
\hline Ethyl hexanoate & ND & - & $6.22 \pm 0.21^{\mathrm{NS}}$ & 1088.00 & $14.89 \pm 0.37^{\mathrm{NS}}$ & 1504.00 & $0.005^{* * *} *$ & $\begin{array}{l}\text { Fruity, apple, } \\
\text { strawberry }\end{array}$ \\
\hline Ethyl octanoate & ND & - & $21.39 \pm 0.78^{\mathrm{NS}}$ & 10255.00 & $28.59 \pm 1.25^{\mathrm{NS}}$ & 15370.00 & $0.002 *$ & $\begin{array}{l}\text { Fruity, floral, } \\
\text { pineapple }\end{array}$ \\
\hline Ethyl nonanoate & ND & - & $1.44 \pm 0.10^{\mathrm{NS}}$ & - & $0.87 \pm 0.04^{\mathrm{NS}}$ & - & - & - \\
\hline Isoamyl acetate & ND & - & $13.71 \pm 0.88^{\mathrm{NS}}$ & 375.67 & $30.56 \pm 3.79^{\mathrm{NS}}$ & 396.00 & $0.03 * * * *$ & $\begin{array}{l}\text { Fruity, banana } \\
\text { sweet }\end{array}$ \\
\hline Isobutyl acetate & ND & - & $1.04 \pm 0.16^{\mathrm{NS}}$ & 0.65 & $0.96 \pm 0.08^{\mathrm{NS}}$ & 0.60 & $1.6^{* *}$ & $\begin{array}{c}\text { Fruity, banana } \\
\text { sweet }\end{array}$ \\
\hline Sum & ND & & $62.46 \pm 1.18^{\mathrm{NS}}$ & & $95.50 \pm 4.59^{\mathrm{NS}}$ & & & \\
\hline \multicolumn{9}{|l|}{ Alcohols } \\
\hline Ethanol & $12.38 \pm 2.45^{\mathrm{c}}$ & - & $160.89 \pm 1.89^{\mathrm{a}}$ & - & $146.98 \pm 3.22^{\mathrm{b}}$ & - & - & - \\
\hline Isobutanol & ND & - & $6.62 \pm 0.08^{\mathrm{NS}}$ & 0.14 & $9.37 \pm 0.58^{\mathrm{NS}}$ & 0.07 & $40^{*}$ & Fruity, banana \\
\hline Isoamyl alcohol & ND & - & $76.76 \pm 3.77^{\mathrm{NS}}$ & 1.65 & $61.67 \pm 2.84^{\mathrm{NS}}$ & 1.32 & $30 *$ & $\begin{array}{l}\text { Banana, sweet, } \\
\text { rancid, rubber }\end{array}$ \\
\hline $\begin{array}{l}\gamma \text {-Methylmercaptopropyl } \\
\text { alcohol }\end{array}$ & ND & - & $0.55 \pm 0.10^{\mathrm{NS}}$ & - & $0.96 \pm 0.13^{\mathrm{NS}}$ & - & - & - \\
\hline 2-Phenylethanol & ND & - & $15.90 \pm 0.34^{\mathrm{NS}}$ & 1.47 & $24.87 \pm 1.12^{\mathrm{NS}}$ & 1.55 & $10^{*}$ & $\begin{array}{l}\text { Floral, rose, } \\
\text { sweet }\end{array}$ \\
\hline Sum & $12.38 \pm 2.45^{\mathrm{c}}$ & & $260.72 \pm 2.22^{\mathrm{a}}$ & & $243.85 \pm 2.38^{\mathrm{b}}$ & & & \\
\hline \multicolumn{9}{|l|}{ Miscellaneous } \\
\hline$\beta$-Ocimene & $0.39 \pm 0.13^{c}$ & - & $10.65 \pm 0.36^{\mathrm{a}}$ & - & $5.71 \pm 0.45^{\mathrm{b}}$ & - & - & - \\
\hline$\alpha$-Ocimene & ND & - & $0.90 \pm 0.05^{\mathrm{NS}}$ & - & $0.58 \pm 0.02^{\mathrm{NS}}$ & - & - & - \\
\hline Styrene & ND & - & $2.13 \pm 0.09^{\mathrm{NS}}$ & - & $3.63 \pm 0.45^{\mathrm{NS}}$ & - & - & - \\
\hline Linalool & ND & & $1.29 \pm 0.06^{\mathrm{NS}}$ & 86.00 & $0.74 \pm 0.07^{\mathrm{NS}}$ & 49.33 & $0.015^{*}$ & $\begin{array}{c}\text { Floral, herbal, } \\
\text { rosewood }\end{array}$ \\
\hline $\begin{array}{l}\text { Methyl N- } \\
\text { hydroxybenzene- } \\
\text { carboximidate. }\end{array}$ & ND & - & $2.10 \pm 0.06^{\mathrm{a}}$ & - & $1.69 \pm 0.02^{\mathrm{b}}$ & - & - & - \\
\hline Acetic acid & $0.69 \pm 0.15^{\mathrm{c}}$ & & $8.56 \pm 0.06^{\mathrm{a}}$ & 0.04 & $7.54 \pm 0.17^{\mathrm{b}}$ & 0.04 & $200^{*}$ & Vinegar \\
\hline 2,3-Butanediol & $0.18 \pm 0.10$ & - & ND & - & ND & - & - & - \\
\hline Sum & $1.27 \pm 0.27^{\mathrm{c}}$ & & $25.62 \pm 0.30^{\mathrm{a}}$ & & $19.89 \pm 0.90^{\mathrm{b}}$ & & & \\
\hline Total & $13.65 \pm 0.10^{\mathrm{c}}$ & & $348.81 \pm 2.86^{\mathrm{b}}$ & & $359.23 \pm 6.04^{\mathrm{a}}$ & & & \\
\hline
\end{tabular}

a,b,c, d Statistical analysis ANOVA $(\mathrm{n}=3)$ at $95 \%$ confidence level with same letters indicating no significant difference.

ND, not detected; NS, not significant; OAVs, Odor activity values; * Murnane et al. (2013); ** Cullere et al. (1997); *** Chen and Liu (2014); **** Chen and Liu (2016); ***** Casassa et al. (2019)

Principal component analysis of volatile compounds in the mixed juices of cashew apple and longan fermented with two $S$. cerevisiae yeasts and Odor activity values

The odor activity values (OAVs) are important for food products, which indicates the contribution of volatile compounds to the aroma. OAVs of the odorants were calculated using the total concentration and the threshold concentration (Cheng et al., 2015). As shown in Table 1 , although a total of 21 volatile compounds were detected in the wines. Generally, volatile aromatic compounds with the OAVs level greater than 1 may potentially contribute to the distinctive aroma of a wine. (Chen and Liu, 2014). Table 1 indicates that seven aroma compounds with OAVs greater than 1 were detected in the two fermentation of wines. As seen in Table 1, seven odorants were selected and quantified: ethyl hexanoate, ethyl octanoate, ethyl decanoate, isoamyl acetate, isoamyl alcohol, 2-phenylethanol, and linalool. Ethyl octanoate had the highest total OAV value, followed by ethyl hexanoate, isoamyl acetate, linalool, ethyl decanoate, isoamyl alcohol, and 2 phenylethanol, respectively (Table 1). This finding was in agreement with previous research which demonstrated that the lychee wines had higher OAVs values of ethyl octanoate, and ethyl hexanoate, ethyl octanoate (Chen and Liu, 2014, 2016). Ethyl hexanoate had a high OAVs value. Therefore, ethyl hexanoate is an important volatile compound in four wine varieties (Cheng $\boldsymbol{e t ~ a l . , ~ 2 0 1 5 ) , ~ a s ~}$ was also confirmed by Sanoppa et al. (2019). The OAVs of ethyl hexanoate, ethyl octanoate, ethyl decanoate, isoamyl acetate, and 2-phenylethanol in the $S$. cerevisiae EC1118 culture were higher than in strain $\mathrm{C} 12$, but there was no significant difference. Regarding these results of this research, there were responsible for the fruity and floral aroma in the mixed fruit wines. Principal component analysis was applied to compare the differences among different fermentations and the volatile compounds of each wine. As shown in Figure 2, the first two principle components accounting for $100 \%$ of the overall variance, with $\mathrm{PC} 1$ contributed to $100 \%$ of the overall variance. The wines obtained from 
both the S. cerevisiae strain C12 and EC1118 cultures could be clearly separated. As a result, the wine cultured with yeast strain EC1118 had higher diversified aroma profiles. The mixed juices cultured with yeast EC1118 was associated with the positive part of PC1, and had high levels of ethyl octanoate, ethyl hexanoate, Isoamyl acetate, ethyl decanoate, and 2-phenylethanol. The fermented wine by yeast $\mathrm{C} 12$ was exhibited in the negative part of $\mathrm{PC} 1$, which was related to higher contents of linalool and isoamyl alcohol.

(A)

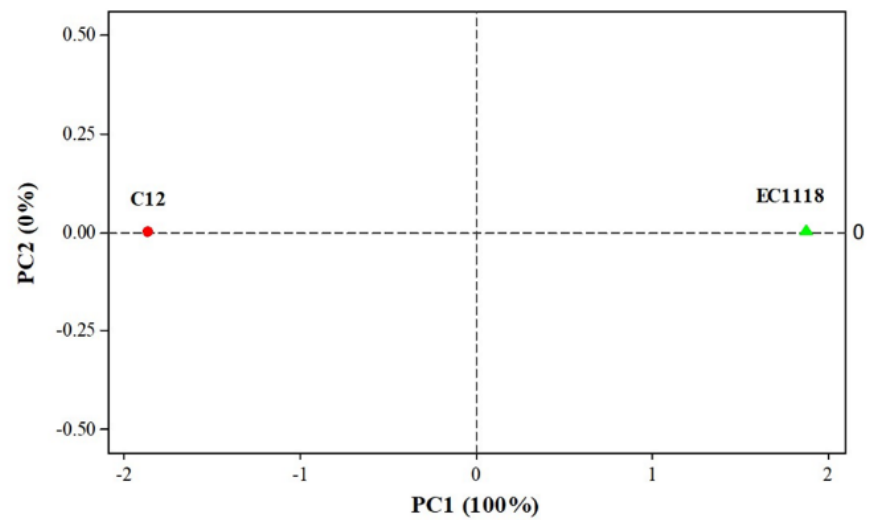

(B)

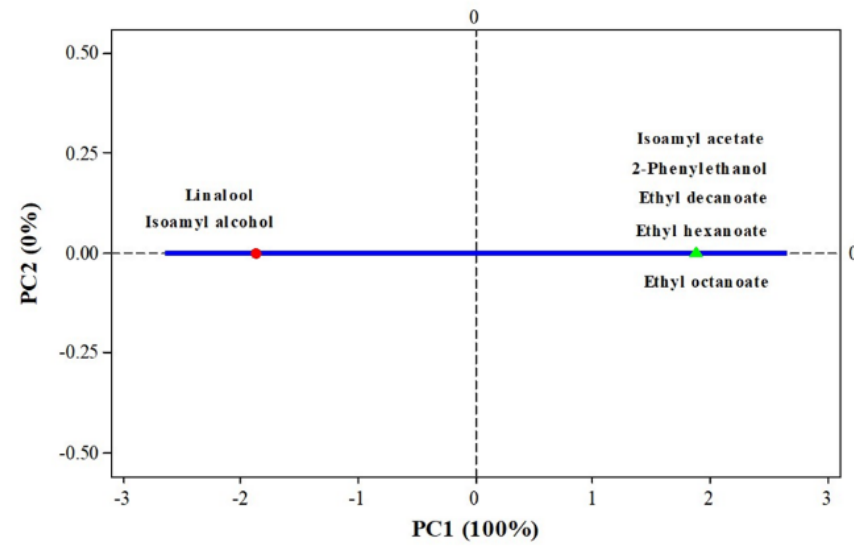

Figure 2 The principal component analysis (PCA) of mixed juice of cashew apple and longan fermented with $S$. cerevisiae $\mathrm{C} 12$ and EC1118.

The PCA score plots of $S$. cerevisiae strains (A), Biplot of the PCA between $S$. cerevisiae strains and volatile compounds in wines (B).

Influence of different inoculation yeast strains on Odor activity values of the mixed juices of cashew apple and longan

As a result, seven odorants had OAVs higher than 0.1 (ethyl octanoate, ethyl hexanoate, isoamyl acetate, linalool, ethyl decanoate, isoamyl alcohol, and 2 phenylethanol) (Table 1). The odorants could be defined as an odor group of aromatic series, including the floral, fruity, sweet, and chemical odors groups, as follows: the floral odor (2-phenylethanol, ethyl octanoate, and ethyl decanoate); the fruity odor (isoamyl alcohol, ethyl decanoate, ethyl hexanoate, isoamyl acetate, and ethyl octanoate); the sweet odor (isoamyl acetate, 2-phenylethanol, and isoamyl alcohol); and the chemical odor (isoamyl alcohol) (Zhang $\boldsymbol{e t}$ al., 2018). As shown in Figure 3, the fruity odor was prominent in the wines, followed by the floral, sweet, and chemical odors, respectively. The ethyl ester of straight-chain fatty acids (ethyl octanoate, ethyl hexanoate, and ethyl decanoate) can provide pleasant fruity and floral odors in the wines (Englezos et al., 2018) The strain EC1118 possessed a higher level of fruity, floral, sweet, and chemical odors in the wines. The chemical odor had a lower concentration than 5 . The results demonstrated that the desired aromatic quality of the mixed fruit juices was fermented by S. cerevisiae EC1118.

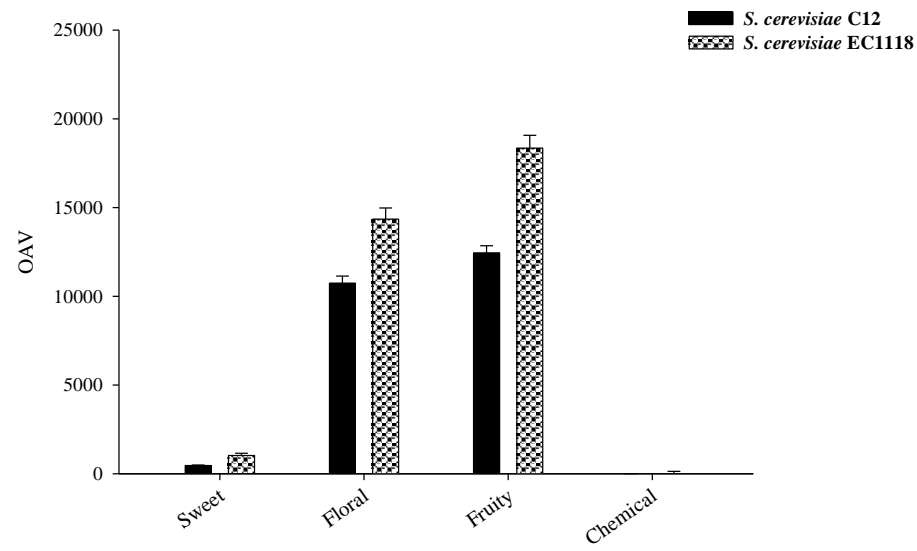

Figure 3 Aroma groups in mixed of cashew apple and longan wines fermented with $S$. cerevisiae $\mathrm{C} 12$ and $\mathrm{EC} 1118$.

\section{Sensory analysis}

We conducted a preference testing for color, clarity, flavor, taste, and overall acceptance (Table 2). There were no significant differences $(p<0.05)$ in terms of these categories among the mixed fruit wines. However, the commercial wine presented significantly higher color, clarity, flavor, taste, and overall acceptance than the mixed fruit wines. The attributes of commercial wines like color, clarity, taste, flavor, and overall acceptance were scored at roughly 8.0 ("like very much"). However, the panelists rated color, clarity, flavor, taste, and overal acceptance scores 6.0-7.3 ("like slightly" - "like moderately"), possibly because the cashew wine had a high tannin content, which imparted a somewhat astringent flavor, as was also confirmed by Mohanty $\boldsymbol{e t}$ al. (2006) in cashew apple wines. Furthermore, ethyl octanoate, isoamyl acetate, and isoamyl alcohol were found in the mixes of cashew apple and longan wines, and were also responsible for a fruity flavor (Zhang $\boldsymbol{e t}$ al., 2018). Nevertheless, the mixed fruit wines were acceptable to all the panelists. Their overall acceptance score was "like slightly." Similar research was reported by Mohanty et al. (2006), who found the panelists accepted the cashew apple juice fermented with $S$. cerevisiae

Table 2 Sensory evaluation of the mixed of cashew and longan wines and commercial wine

\begin{tabular}{lccc}
\hline \multirow{2}{*}{ Attributes } & $\begin{array}{c}\text { Commercial } \\
\text { wine }\end{array}$ & \multicolumn{2}{c}{$\begin{array}{c}\text { The mixed of cashew apple and longan } \\
\text { wines }\end{array}$} \\
\cline { 3 - 4 } & & S. cerevisiae $\mathrm{C} 12$ & $\begin{array}{c}\text { S. cerevisiae } \\
\text { EC1118 }\end{array}$ \\
\hline Color & $8.47 \pm 0.51^{\mathrm{a}}$ & $7.23 \pm 0.86^{\mathrm{b}}$ & $7.27 \pm 0.64^{\mathrm{b}}$ \\
Clarify & $8.43 \pm 0.50^{\mathrm{a}}$ & $6.27 \pm 0.58^{\mathrm{b}}$ & $6.30 \pm 0.65^{\mathrm{b}}$ \\
Flavor & $8.23 \pm 0.50^{\mathrm{a}}$ & $6.37 \pm 0.67^{\mathrm{b}}$ & $6.33 \pm 0.48^{\mathrm{b}}$ \\
Taste & $8.43 \pm 0.50^{\mathrm{a}}$ & $6.07 \pm 0.69^{\mathrm{b}}$ & $6.10 \pm 0.55^{\mathrm{b}}$ \\
Overall & $8.40 \pm 0.67^{\mathrm{a}}$ & $6.27 \pm 0.78^{\mathrm{b}}$ & $6.37 \pm 0.85^{\mathrm{b}}$ \\
\hline
\end{tabular}

\section{CONCLUSION}

Cashew apple and dried longan are important economic crops in Thailand and are also an interesting option to produce wine because they contain a wide variety of nutrients. Our purpose was to investigate the effects of the culture of two yeast strains of $S$. cerevisiae on the microbiological, chemical, and volatiles content in the mixed fruit wines. These results demonstrated that both the $S$. cerevisiae strain $\mathrm{C} 12$ and EC1118 can increase the aroma intensity and complexity of the wines. Seven odorants had OAVs higher than 1 , which can make an active contribution to the wine's aroma. The mixed juices of cashew apple and longan fermented with the $S$. cerevisiae strain EC1118 significantly produced the highes total volatile contents, and we also found a higher total level of esters, but there was no statistically significant difference when compared with the fermentation inoculated with $S$. cerevisiae strain $\mathrm{C} 12$. On the contrary, $S$. cerevisiae $\mathrm{C} 12$ produced significantly higher concentrations of total alcohol and miscellaneous elements than with $S$. cerevisiae EC1118. As the result, the volatile compounds in the mixed fruit wines correlated with the panelists' sensory evaluations. Those evaluations indicate that the mixed of cashew apple and longan wines imparted the characteristic cashew apple and dried longan and astringency. Therefore, the panelists rated their overall acceptance of the product as "like slightly." However, both the mixed juices of cashew apple and longan cultured with yeast strain $\mathrm{C} 12$ and EC1118 exhibited the highest of the fruity odorants group in the mixed fruit wines. The $S$. cerevisiae EC1118 culture positively affected the aroma characteristic. 
Acknowledgment: This research was funded by King Mongkut's University of Technology North Bangkok. Contract no. KMUTNB-GEN-57-40.

\section{REFERENCES}

Agrawal, N., Minj, D. K., \& Rani, K. (2015). Estimation of Total Carbohydrate Present In Dry Fruits. IOSR Journal of Environmental Science, Toxicology and Food Technology, 1(6), 24-27.

AOAC. (2005). Official Methods of Analysis of AOAC International. Maryland, USA: Association of Official Analytical Chemists.

Balogun, M. A., Abiodun, O. A., Kolawole, F. L., Kayode, R. M. O., \& Olushola , O. E. (2017). Physicochemical and sensory properties of blends of pineapplecarrot wine. Journal of microbiology, biotechnology and food science, 7(3), 306311 doi:https://doi.org/10.15414/jmbfs.2017/18.7.3.306-311

Carrau, F. M., Medina, K., Farina, L., Farina, E., Henschke, P. A., \& Dellacassa, E. (2008). Production of fermentation aroma compounds by Saccharomyces cerevisiae wine yeasts: ejects of yeast assimilable nitrogen on two model strains. FEMS Yeast Research, 8(7), 1196-1207. doi:https://doi.org/10.1111/j.15671364.2008.00412.x

Casassa, L. F., Sari, S. E., Bolcato, E. A., Diaz-Sambueza, M. A., Catania, A. A., Fanzone, M. L., Raco, F., \& Barda, N. (2019). Chemical and sensory effects of cold soak, whole cluster fermentation, and stem additions in pinot noir wines American Journal of Enology and Viticulture, 70(1), 19-33. doi https://doi.org/10.5344/ajev.2018.18014

Chang, C. Y., Chang, C. H., Yu, T. H., Lin, L. Y., \& Yen, Y. H. (1998). The effect of drying treatment on the flavour and quality of longan fruit. Food Flavours: Formation Analysis and Packaging Influences, 40, 353-346. doi:https://doi.org/10.1016/S0167-4501(98)80059-9

Chen, D., \& Liu, S. Q. (2014). Chemical and volatile composition of lychee wines fermented with four commercial Saccharomyces cerevisiae yeast strains. International Journal of Food Science and Technology, 49, 521-530. doi:https://doi.org/10.1111/ijfs.12332

Chen, D., \& Liu, S. Q. (2016). Impact of simultaneous and sequential fermentation with Torulaspora delbrueckii and Saccharomyces cerevisiae on non-volatiles and volatiles of lychee wines. LWT-Food Science and Technology, 65, 53-61. doi:https://doi.org/10.1016/j.lwt.2015.07.050

Cheng, G., Liu, Y., Yue, T. X., \& Zheng, Z. W. (2015). Comparison between aroma compounds in wines from four Vitis vinifera grape varieties grown in different shoot positions. Food Science and Technology, 35, 237-246. doi:http://dx.doi.org/10.1590/1678-457X.6438

Cullere, L., Escudero, A., Cacho, J., \& Ferreira, V. (2004). Gas chromatography-olfactometry and chemical quantitative study of the aroma of six premium quality spanish aged red wines. Journal of Agricultural and Food Chemistry, 52, 1653-1660. doi: https://doi.org/10.1021/jf0350820

Deenanath, E. D., Rumbold, K., Daramola, M., Falcon, R., \& Iyuke, S. (2015) Evaluation of Physicochemical Properties of South African Cashew Apple Juice $\begin{array}{lllll}\text { as a Biofuel } \quad \text { Feedstock. } & \text { Scientifica, } & \text { 2015, }\end{array}$ doi:http://dx.doi.org/10.1155/2015/764196

Dickinson, J. R., \& Norte, V. (1993). A study of branched-chain amino acid aminotransferase and isolation of mutations affecting the catabolism of branchedchain amino acids in Saccharomyces cerevisiae. Febs Letters, 326(1-3), 29-32. doi:https://doi.org/10.1016/0014-5793(93)81754-n

Englezos, V., Rantsiou, K., Cravero, F., Torchio, F., Giacosa, S., Ortiz-Julien, A., Gerbi, V., Rolle, L., \& Cocolin, L. (2018). Volatile profiles and chromatic characteristics of red wines produced with Starmerella bacillaris and Saccharomyces cerevisiae. Food Research International,, 109, 298-309. doi:https://doi.org/10.1016/j.foodres.2018.04.027

Etschmann, M. M. W., Kotter, P., Kötter, J., Bluemke, W., Entian, K. D., \& Schrader, J. (2008). Production of the aroma chemicals 3-(methylthio)-1propanol and 3-(methylthio)-propylacetate with yeasts. Applied Microbiology Biotechnology, 80, 579-587. doi:https://doi.org/10.1007/s00253-008-1573-4 Garruti, D. S., Franco, M. R. B., Silva, M. P. A. A. P., Janzantti, N. S., \& Alves, G. L. (2006). Assessment of aroma impact compounds in a cashew apple-based alcoholic beverage by GC-MS and GC-olfactometry. LWT - Food Science and Technology, 39(4). doi:https://doi.org/10.1016/j.lwt.2005.02.006

Gawankar, M. S., Salvi, B. R., Pawar, C. D., Khanvilkar, M. H., Salvi, S. P., Dalvi, N. V., Malshe, K. V., Kadam, D. S., Saitwal, Y. S., \& Haldankar, P. M. (2018). Technology Development For Cashew Apple Processing In Konkan Region - A Review. Advanced Agricultural Research \& Technology Journal, 11(1), 40-47.

Guth, H. (1997). Quantitation and Sensory Studies of Character Impact Odorants of Different White Wine Varieties. Journal of Agricultural and Food Chemistry, 45, 3027-3032. doi:https://doi.org/10.1021/jf970280a

Ilc, T., Werck-Reichhart, D., \& Navrot, N. (2016). Meta-analysis of the core aroma components of grape and wine aroma. Frontiers in Plant Science, 7, 1-15. doi:https://dx.doi.org/10.3389\%2Ffpls.2016.01472

Lee, P. R., Ong, Y. L., Yu, B., Curran, P., \& Liu, S. Q. (2010). Evolution of volatile compounds in papaya wine fermented with three Willopsis saturnus yeasts. International Journal of Food Science and Technology, 45, 2032-2041. doi:https://doi.org/10.1111/j.1365-2621.2010.02369.x
Kaewbutra, S., Wattanathorn, J., Thukham-mee, W., Muchimapura, S., \& TongUn, T. (2016). Cashew Apple Juice Improves Physical Fitness and Oxidative Stress Status in the Middle-Aged and Elderly Volunteers. The Journal of Medical Association of Thailand, 99(8), 161-172.

Lee, P. R., Toh, M., Yu, B., Curran, P., \& Li, S. Q. (2013). Manipulation of volatile compound transformation in durian wine by nitrogen supplementation. International Journal of Food Science and Technology, 48(3), 650-662. doi:https://doi.org/10.1111/ijfs.12012

Lilly, M., Lambrechts, M. G., \& Pretorius, I. S. (2000). Effect of increased yeast alcohol acetyltransferase activity on flavor profiles of wine and distillates. Appl Environ Microbiol, 66(2), 744-753. doi:https://doi.org/10.1128/AEM.66.2.744$\underline{753.2000}$

Mohunty, S., Ray, P., Swain , M. R., \& Ray, R. C. (2006). Fermentation of cashew (Anacardium occidentale L.) "apple" in to wine. Journal of Food Processing and Preservation, 30, 314-322. doi:https://doi.org/10.1111/j.17454549.2006.00067.x

Murnane, S. S., Lehocky, A. H., \& Owens, P. D. (2013). Odor Thresholds for Chemicals with Established Occupational Health Standards (Vol. 2). U.S.A.: The American Industrial Hygiene Association (AIHA®).

Okeke, B. C., Agu, K. C., Uba, P. O., Awah, N. S., Anaukwu, C. G., Archibong, E. J., Uwanta, L. I., Ezeneche, J. N., Ezenwa, C. U., \& Orji, M. U. (2015). Wine Production from Mixed Fruits (Pineapple and Watermelon) Using High Alcohol Tolerant Yeast Isolated from Palm Wine. Universal Journal of Microbiology Research, 3(4), 41-45. doi:https://doi.org/10.13189/ujmr.2015.030401

Parapouli, M., Vasileiadis, A., Afendra, A. S., \& Hatziloukas, E. (2020) Saccharomyces cerevisiae and its industrial applications. AIMS Microbiology, 6(1), 1-31. doi:https://doi.org/10.3934/microbiol.2020001

Procopio, S., Sprung, P., \& Becker, T. (2015). Effect of amino acid supply on the transcription of flavour-related genes and aroma compound production during lager yeast fermentation. LWT - Food Science and Technology, 63, 289-297. doi:https://doi.org/10.1016/j.lwt.2015.03.007

Ray, R. C., Panda, S. K., Swain , M. R., \& Sivakumar, P. S. (2011). Proximate composition and sensory evaluation of anthocyanin-rich purple sweet potato (Ipomoea batatas L.) wine. International Journal of Food Science and Technology, 47(3), 452-458. doi:https://doi.org/10.1111/j.13652621.2011.02861.x

Saerens, S. M. G., Delvaux, F. R., Verstrepen, K. J., \& Thevelein, J. M. (2010). Production and biological function of volatile esters in Saccharomyces $\begin{array}{llll}\text { cerevisiae. } & \text { Microbial } & \text { Biotechnology, } & \text { 3, }\end{array}$ doi:https://doi.org/10.1111/j.1751-7915.2009.00106.x

Saerens, S. M. G., Verstrepen, K. J., Van Laere, S. D. M., Voret, A. R. D., Dijck, P. V., Delvaux, F. R., \& Thevelein, J. M. (2005). The Saccharomyces cerevisiae EHT1 and EEB1 Genes Encode Novel Enzymes with Medium-chain Fatty Acid Ethyl Ester Synthesis and Hydrolysis Capacity. The journal of biological chemistry, 281, 4446-4456. doi:https://doi.org/10.1074/jbc.M512028200

Sanoppa, K., Huang, T. C., \& Wu, M. C. (2019). Effects of Saccharomyces cerevisiae in association with Torulaspora delbrueckii on the aroma and amino acids in longan wines. Food Science \& Nutrition, 7(9), 2817-2826. doi:https://doi.org/10.1002/fsn3.1076

Sanoppa, K., Huang, T. C., \& Wu, M. C. (2020). Effects of the Mixed Fermentation of Torulaspora delbrueckii and Saccharomyces cerevisiae on the Non-Volatile and Volatile Compounds and the Antioxidant Activity in Golden Dried Longan Wine Microbiology Biotechnology Letters, 48(1), 1-11. doi:http://dx.doi.org/10.4014/mbl.1907.07006

Swiegers, J. H., Bartowsky, E. J., Henschke, P. A., \& Pretorius, I. S. (2005). Yeast and bacterial modulation of wine aroma and flavour. Australian Journal of Grape and Wine Research, 11, 139-173. doi:https://doi.org/10.1111/j.17550238.2005.tb00285.x

Trinh, T. T. T., Woon, W. Y., Yu, B., \& Liu, S. Q. (2010). Effect of L-isoleucine and L-phenylalanine Addition on Aroma Compound Formation During Longan Juice Fermentation by a Co-culture of Saccharomyces cerevisiae and Williopsis saturnus. South African Journal for Enology and Viticulture, 31, 116-124. doi:https://doi.org/10.21548/31-2-1408

Walker, G. M., \& Stewart, G. G. (2016). Saccharomyces cerevisiae in the Production of Fermented Beverages. Beverages, 2(30), 1-12. doi: https://doi.org/10.3390/beverages2040030

Zhang, B. Q., Luan, Y., Duan, C. Q., \& L., Y. G. (2018). Use of Torulaspora delbrueckii Co-fermentation with two Saccharomyces cerevisiae strains with different aromatic characteristic to improve the diversity of red wine aroma $\begin{array}{llll}\text { profile. Frontiers in } & \text { Microbiology, }\end{array}$ doi:https://doi.org/10.3389/fmicb.2018.00606

Zhang, J., Zhang, C., Qi, Y., Dai, L., Ma, H., Guo, X., \& Xiao, D. (2014). Acetate ester production by Chinese yellow rice wine yeast overexpressing the alcohol acetyltransferase-encoding gene ATF2. Genetics and Molecular Research, 4, 9735-9746. doi:https://doi.org/10.4238/2014. 